\title{
Avaliação dos atributos da Atenção Primária à Saúde na perspectiva dos idosos ${ }^{a}$
}

\author{
Assessment of the attributes of Primary Health Care from the perspective of older adults \\ Evaluación de los atributos de la Atención Primaria a la Salud desde la perspectiva de los adultos \\ mayores
}

Rosângela Guerino Masochini ${ }^{1,2}$ (D) Sheila Nascimento Pereira de Farias ${ }^{1}$ (D)

Ana Inês Sousa ${ }^{1}$ (D)

1. Universidade Federal do Rio de Janeiro. Rio de Janeiro, RJ, Brasil.

2. Universidade Federal de Mato Grosso. Sinop, MT, Brasil.
Autor correspondente:

Rosângela Guerino Masochini.

E-mail: rguerino320@hotmail.com

Recebido em 27/10/2020.

Aprovado em 29/09/2021.

DOI:https://doi.org/10.1590/2177-9465-EAN-2020-0433

\section{RESUMO}

Objetivo: avaliar os atributos da Atenção Primária à Saúde na perspectiva dos idosos, na cidade de Sinop em Mato Grosso. Método: pesquisa avaliativa, de abordagem quantitativa e delineamento transversal. Utilizou-se o instrumento Primary Care Assessment Tool para entrevista aos 345 idosos. Após entrevista com o instrumento Primary Care Assessment Tool -Brasil, versão usuários, estimou-se o nível de qualidade dos atributos essenciais e derivados sendo considerado satisfatório o escore $\geq 6,6$ e insatisfatório <6,6. A análise foi feita no programa SPSS, versão 19.0. Resultados: os atributos Longitudinalidade, Coordenação e Integralidade obtiveram avaliações satisfatórias. O Acesso de Primeiro Contato obteve a pior avaliação na perspectiva dos idosos. Conclusão: verifica-se que o acesso de primeiro contato constitui uma barreira a ser transposta na busca de atender às necessidades dos idosos.

Palavras-chave: Avaliação em Saúde; Idoso; Estratégia de Saúde da Família; Saúde Pública.

\section{ABSTRACT}

Objective: to assess the attributes of Primary Health Care from the perspective of older adults in the municipality of Sinop, Mato Grosso. Method: this is an evaluative research study with a cross-sectional design. The data were collected using the Primary Care Assessment Tool for interviews with 345 older adults. After the interviews, with the Primary Care Assessment Tool - Brazil, users' version, the quality level was estimated for the essential and derivative attributes with satisfactory scores $\geq 6.6$, and unsatisfactory scores < 6.6. The analysis was performed using the SPSS software, version 19.0. Results: the Logitudinality, Coordination and Completeness attributes obtained satisfactory assessments. First Contact Access obtained the worse assessment from the perspective of older adults. Conclusion: it is verified that the first contact access constitutes a barrier to be overcome in seeking to meet the older adults' needs.

Keywords: Health Evaluation; Aged; Family Health Strategy; Public Health.

\section{RESUMEN}

Objetivo: evaluar los atributos de la Atención Primaria a la Salud desde la perspectiva de los adultos mayores en la ciudad de Sinop en Mato Grosso. Método: investigación evaluativa, de enfoque cuantitativo y delineamiento transversal. Se utilizó e instrumento Primary Care Assessment Tool en la entrevista a 345 adultos mayores. Después de la entrevista con el instrumento Primary Care Assessment Tool-Brasil, versión usuarios, se estimó el nivel de calidad de los atributos esenciales y derivados, y se consideró satisfactorio cuando la puntuación era $\geq 6,6$ e insatisfactorio el puntaje $<6,6$. El análisis se llevó a cabo en e programa SPSS, versión 19.0. Resultados: los atributos Longitudinalidad, Coordinación e Integralidad obtuvieron evaluaciones satisfactorias. El Acceso de Primer Contacto obtuvo la peor evaluación en la perspectiva de los adultos mayores. Conclusión se constató que el acceso de primer contacto constituye una barrera a ser traspuesta en el intento de satisfacer las necesidades de los adultos mayores.

Palabras clave: Atención Primaria de la Salud; Evaluación en Salud; Estrategia de Salud Familiar; Adulto Mayor; Salud Pública. 


\section{INTRODUÇÃO}

A longevidade é um fenômeno mundial e, juntamente com a queda da fecundidade, ocasiona o envelhecimento da população no planeta. Em outros países, como no caso o Brasil, os impactos oriundos do envelhecimento da nação são ainda maiores devido ao rápido processo de senescência do país e, consequentemente, a melhoria da expectativa média de vida levará a população atual a encontrar a velhice ${ }^{1}$.

O envelhecimento da população brasileira traz diversos desafios, principalmente nos serviços de saúde, que realizam atividades de acompanhamento de doenças crônicas, em destaque, a Atenção Primária à Saúde (APS), que visa a promoção, prevenção, tratamento e reabilitação da saúde ${ }^{2}$ integrando, atualmente, uma área de interesse mundial em função da posição estratégica na prevenção de agravos e promoção da saúde da população ${ }^{3}$.

Starfiel ${ }^{4}$ estabeleceu qualidades próprias à APS, chamadas atributos essenciais e derivados. Estes atributos qualificam as ações em Atenção Primária à Saúde, aumentando seu poder de interação com os indivíduos e com a comunidade. E, para avaliação destes atributos, desenvolveu o instrumento denominado de Primary Care Assessment Tool que apresenta originalmente versões destinadas a crianças, adultos, profissionais de saúde e gestores ${ }^{2,5}$.

Os atributos essenciais são assim chamados, pois o serviço de atenção básica, dirigido à população geral só pode ser considerado provedor de atenção primária quando há a sua presença. Eles qualificam as ações em atenção primária à saúde, aumentando seu poder de interação com os indivíduos e com a comunidade. São quatro os atributos essenciais: Acesso de Primeiro Contato, Longitudinalidade, Coordenação e Integralidade. Os derivados são: orientação familiar, orientação comunitária e competência cultural. Esses atributos podem ser analisados individualmente embora apresentarem relação na prática clínica ${ }^{4,5,6}$.

A identificação destes atributos é importante para definir se o serviço de saúde está realmente embasado em Atenção Primária à Saúde ${ }^{4,5}$.

Avaliar a qualidade do serviço ofertado, o acesso ao primeiro contato dos usuários, a disponibilidade da equipe multiprofissional em prestar atendimento, a integração dos cuidados e das necessidades de cada cliente e a orientação à integralidade do atendimento em todas as esferas disponibilizadas nos serviços de saúde possibilita, em sua totalidade, a identificação de qualidade referente a toda equipe inserida neste nível de atenção ${ }^{1}$.

A estrutura e organização dos serviços, interferem na assistência ofertada aos usuários e principalmente aos idosos.

A capacidade de enfrentamento desses desafios, vale tanto para os pacientes quanto para os usuários, para a promoção do bem-estar e da qualidade de vida dos usuários ${ }^{6}$.

O objetivo desta pesquisa foi avaliar os atributos essenciais e derivados da Atenção Primária à Saúde na perspectiva dos idosos.

\section{MÉTODO}

Trata-se de uma pesquisa avaliativa, descritiva do tipo transversal, com abordagem quantitativa e é um recorte da pesquisa intitulada "Avaliação da qualidade da Atenção Primária à Saúde em um município da região da Amazônia Legal" aprovada pelo Comitê de Ética "Humanidades" da Universidade Federal do Mato Grosso (UFMT), número 1450546.

Foi realizada no município de Sinop, estado de Mato Grosso o qual possui 132.934 habitantes $^{7}$ e 20 equipes Estratégia Saúde da Família.

Para o estudo, foi considerada uma amostra "finita" de 3.349 usuários idosos cadastrados nas 15 Unidades de Saúde da Família existentes no município. Utilizando a fórmula para amostra finita, foi estimada uma amostra total de 345. Na busca de selecionar os idosos de forma proporcional em cada unidade de saúde, utilizou-se a amostra estratificada. Em cada estrato amostral, os idosos foram selecionados de forma aleatória utilizando a Ficha de Cadastro da Família.

O instrumento utilizado foi Primary Care Assessment Tool, versão usuários, e totaliza 87 itens divididos em 10 componentes relacionados aos atributos da APS. O “Grau de Afiliação" utilizado no cálculo dos escores, não é considerado atributo.

Para o cálculo dos escores dos atributos da APS, utilizou-se média aritmética simples e a seguinte formula (Escore obtido 1) $x 10 / 3$ foi seguida para transformar os valores das respostas em escala de 0 a $10^{2,4}$.

Em conformidade com as normas citadas, com o objetivo de facilitar a interpretação da grandeza dos valores, foram tomados como referência os valores estabelecidos no instrumento original, os quais foram utilizados em estudos realizados com a aplicação do PCATool-Brasil versão usuários, conforme descrito a seguir: Escores $\geq 6,6$ e atributos equivalentes ao valor três ou mais na escala Likert, foram considerados satisfatório; e Escores $<6,6 \mathrm{e}$ atributos correspondentes a valores inferiores a 3 na escala Likert, classificados em insatisfatório ${ }^{5,6}$.

Para a análise dos dados, criou-se um banco de dados no programa Microsoft Office Excel 2010, no qual os dados foram compilados e agrupados de acordo com os blocos de perguntas dos instrumentos utilizados na coleta de dados e as características sociodemográficas dos idosos. A seguir, os dados coletados foram transcritos para o programa SPSS, versão 19.0 para Windows, para a execução do processamento e a submissão das análises. A análise estatística descritiva foi realizada de forma que as variáveis categóricas foram descritas por frequências absolutas, porcentagens e as variáveis quantitativas, por meio de média, desvio padrão e teste de Mann-Whitney.

\section{RESULTADOS}

Participaram 345 idosos vinculados a 20 equipes de saúde, todas são organizadas em Estratégia de Saúde da Família e possuem a equipe de profissionais de saúde completa (médico, enfermeiro, auxiliar ou técnico de enfermagem, agente comunitário de saúde, cirurgião-dentista, auxiliar ou técnico em saúde bucal) ${ }^{8}$, conforme preconiza a Portaria no 2.488 de outubro de $2011^{9}$.

Em relação ao sexo, apresentou $88,4 \%$ feminino e a média de idade foi de 72,4 anos, variando entre 60 e 90 anos de idade, $46,1 \%$ estão casados, 51,3\% possuem renda de até 1 salário 
mínimo, 53,3\% são aposentados e $84,1 \%$ dos idosos se dizem independentes financeiramente. Em relação aos problemas de saúde, a principal doença referida foi a Hipertensão Arterial Sistêmica (59,7\%).

Em relação ao grau de afiliação, 302 idosos responderam sim nas três perguntas: Há um profissional ou serviço de saúde aonde você geralmente vai quando fica doente ou precisa de conselhos sobre a sua saúde? Há um profissional ou serviço de saúde que o/a conhece melhor como pessoa? Há um profissional ou serviço de saúde que é mais responsável por seu atendimento de saúde? O grau de afiliação dos idosos está centrado no profissional médico'.

A Tabela 1 representa os atributos da APS. Entre os atributos derivados, destaca-se "orientação familiar" $(8,86)$ seguido de "orientação comunitária" (6,73). E, entre os atributos essenciais, teve-se o "acesso-utilização" $(9,69)$, "longitudinalidade" $(8,04)$, "coordenação de cuidados" (8,42), "coordenação de informações" $(8,17)$, "serviços prestados" $(8,30)$ e "serviços disponíveis" $(7,66)$. Estes atributos apresentaram posição de destaque na perspectiva dos idosos, pois apresentaram média $\geq$ a 6,6 sendo classificados em satisfatórios.

O único atributo classificado em insatisfatório foi "acessoacessibilidade" que apresentou o escore $(2,57)$.

$\mathrm{Na}$ Tabela 2, verifica-se que entre os 10 atributos da APS, 9 obtiveram resultados satisfatórios e todos apresentaram diferença estatisticamente significante $(p<0,001)$. Contudo, mesmo entre os idosos que avaliaram a APS em alto escore geral, as condições de acessibilidade foram aquém do necessário, dos 345 idosos

Tabela 1. Escores atribuídos aos atributos da APS conferidos pelos idosos. SINOP, 2016.

\begin{tabular}{lccccc}
\hline \multicolumn{1}{c}{ ATRIBUTOS } & Média & \multicolumn{2}{c}{ Inferior } & Superior & Mediana \\
\hline Grau de afiliação & 3,81 & 3,77 & 3,88 & 4,00 & 0,50 \\
Acesso-utilização & 9,69 & 9,60 & 9,78 & 10,00 & 0,82 \\
Acesso-acessibilidade & 2,57 & 2,49 & 2,64 & 2,50 & 0,69 \\
Longitudinalidade & 8,04 & 7,98 & 8,10 & 8,10 & 0,54 \\
Coordenação de cuidados & 8,42 & 8,24 & 8,59 & 8,33 & 1,66 \\
Coordenação de informações & 8,17 & 8,05 & 8,28 & 7,78 & 1,08 \\
Serviços disponíveis & 7,66 & 7,62 & 7,70 & 7,58 & 0,41 \\
Serviços prestados & 8,30 & 8,26 & 8,35 & 8,46 & 0,42 \\
Orientação familiar & 8,84 & 8,64 & 8,83 & 8,89 & 0,96 \\
Orientação comunitária & 6,73 & 6,64 & 6,83 & 6,67 & 0,87 \\
Essencial & 6,43 & 6,39 & 6,47 & 6,49 & 0,40 \\
Geral & 6,70 & 6,65 & 6,74 & 6,76 & 0,40 \\
\hline
\end{tabular}

Nota: IC - intervalo de confiança de 95\%; DP desvio-padrão.

Tabela 2. Análise da classificação dos escores dos atributos da APS na visão dos idosos. SINOP, 2016.

\begin{tabular}{lccccc}
\hline \multicolumn{1}{c}{ ATRIBUTOS } & $\mathrm{n}$ & $\%$ & $\mathrm{n}$ & $\%$ & $\mathrm{p}$ \\
\hline Grau de afiliação & 6 & 1,7 & 339 & 98,3 & $0,0001^{*}$ \\
\hline Acesso-utilização & 1 & 0,3 & 344 & 99,7 & $0,0001^{*}$ \\
\hline Acesso-acessibilidade & 344 & 99,7 & 1 & 0,3 & $0,0001^{*}$ \\
\hline Longitudinalidade & 3 & 0,9 & 342 & 99,1 & $0,0001^{*}$ \\
\hline Coordenação de cuidados & 9 & 2,6 & 336 & 97,4 & $0,0001^{*}$ \\
\hline Coordenação de informações & 9 & 2,6 & 336 & 97,4 & $0,0001^{*}$ \\
\hline Integralidade de serviços disponíveis & 0 & 0,0 & 345 & 100 & - \\
\hline Integralidade de serviços prestados & 1 & 0,3 & 344 & 99,7 & $0,0001^{*}$ \\
\hline Orientação familiar & 2 & 0,6 & 343 & 99,4 & $0,0001^{*}$ \\
Orientação comunitária & 75 & 21,7 & 270 & 78,1 & $0,0001^{*}$ \\
\hline Escore essencial & 231 & 67,0 & 114 & 33,0 & $0,0001^{*}$ \\
\hline Escore geral & 87 & 25,2 & 258 & 74,8 & $0,0001^{*}$ \\
\hline
\end{tabular}

Nota: $* p \leq 0,05$ diferença estatisticamente significativa na distribuição de proporção pelo teste do Mann-Whitney. Valores $<6,6$ foram classificados com "Insatisfatório", e valores $\geq 6,6$ como Satisfatório. 
apenas $1(0,3 \%)$ idoso classificou o acesso em satisfatório e $344(99,7 \%)$ classificaram o acesso em insatisfatório.

\section{DISCUSSÃO}

Os idosos entrevistados avaliaram o atendimento recebido na atenção primária como sendo parcialmente satisfatório, havendo necessidade de melhoria, principalmente em relação ao escore Essencial $(6,4)$ e Geral $(6,7)$. Isso corrobora os resultados em único estudo anterior, realizado com 100 idosos, em Macaíba, Rio Grande do Norte $^{10}$, que apresentou os seguintes escores Geral $(5,7)$ e Essencial $(6,0)$ ficando distante da nota máxima possível $(10,0)$. Em relação aos estudos realizados em outras faixas etárias, tem-se o estudo realizado em Municípios do Sul de Minas Gerais ${ }^{11}$, com 527 adultos acima de 18 anos e apresentou escore Essencial Adulto $(5,96)$ e escore Geral $(5,92)^{10}$. Sendo as populações comparáveis, os resultados indicam que não existe priorização da assistência ao idoso no município estudado, fato esse que não vem de encontro com a Política Nacional do Idoso que assegura o atendimento preferencial aos serviços de saúde. O primeiro atributo que compõe o instrumento Primary Care Assessment Tool versão usuários é o atributo "acessoutilização" e "acesso-acessibilidade"2. Neste estudo, verificase que o "acesso-acessibilidade" obteve a única classificação insatisfatória. A "acessibilidade" representa a ausência de barreiras organizacionais e físicas para a obtenção da atenção à saúde, como a restrição de dias e horários de funcionamento.

As unidades de saúde no município de Sinop atendem somente durante os dias da semana e em horário limitado, das 7:00 às 11:00 horas e das 13:00 às 17:00 horas. Este horário de atendimento representa séria limitação, sendo que há um intervalo diário descoberto para a prestação de serviço aos idosos e, consequentemente, poderia sobrecarregar o atendimento na única Unidade de Pronto Atendimento (UPA). A mudança de horários, sendo a oferta de atendimento no período noturno e nos finais de semana, poderia contribuir para melhoria desse atributo. A fim de implementar essas mudanças, é necessário investimento financeiro para contratação de profissionais e abertura de novas unidades. Com essas modificações, o serviço de saúde, além de ampliar o acesso aos idosos, poderá trazer melhoria na assistência prestada, principalmente o acolhimento, que possibilita a autonomia, a cidadania e a corresponsabilização na produção do cuidado à saúde ${ }^{8}$.

Em relação ao atributo "longitudinalidade", obteve-se o resultado satisfatório. Um resultado satisfatório nesse atributo sinaliza que existe continuidade do cuidado em relação ao idoso e o serviço de saúde, com construção de vínculo. Resultados positivos foram encontrados em outros estudos, na faixa etária acima de 18 anos e acima de 60 anos ${ }^{9,10}$. O atributo "coordenação" é dividido em coordenação de cuidados e coordenação de informação. Foram avaliados em satisfatórios, apresentando os resultados 8,34 para coordenação de cuidados e 8,17 para coordenação de informações. Os itens pior avaliados foram "contrareferência", "falta à continuidade do cuidado", ou seja, os profissionais encaminham os idosos ao serviço de referência, porém não recebem por escrito qual foi a conduta realizada, o que impossibilita a continuidade do cuidado ao idoso ${ }^{12}$. Em relação à disponibilização do prontuário, os idosos não possuem o conhecimento de que podem consultá-lo. Infere-se a necessidade de orientação aos idosos, pois o prontuário é do paciente e o mesmo tem direito de acesso à cópia, pelo código de defesa do Consumidor, artigo 72 e o Código de Ética Médica na Resolução do Conselho Federal de Medicina nº 1.246/88²,13.

Os idosos entrevistados, de modo geral, avaliaram o atendimento recebido na atenção primária como sendo parcialmente satisfatório, havendo necessidade de melhoria, principalmente em relação ao escore Essencial $(6,4)$ e Geral $(6,7)$. Isso corrobora os resultados em único estudo anterior, realizado com 100 idosos, em Macaíba (RN), que apresentou os seguintes escores Geral $(5,7)$ e Essencial $(6,0)$ ficando distante da nota máxima possível $(10,0)^{9}$. Em relação aos estudos realizados em outras faixas etárias, tem-se o estudo realizado em Municípios do Sul de Minas Gerais, com 527 adultos acima de 18 anos e apresentou escore Essencial Adulto $(5,96)$ e escore Geral $(5,92)^{10}$. Sendo as populações comparáveis, os resultados indicam que não existe priorização da assistência ao idoso no município estudado, fato esse que não vem de encontro com a Politica Nacional do Idoso que assegura o atendimento preferencial aos serviços de saúde ${ }^{9}$.

O primeiro atributo que compõe o instrumento Primary Care Assessment Tool versão usuários é o atributo "acesso-utilização" e "acesso-acessibilidade". Neste estudo, verifica-se que o atributo "acesso-acessibilidade" obteve a única classificação insatisfatória

A "acessibilidade" representa a ausência de barreiras organizacionais e físicas para a obtenção da atenção à saúde ${ }^{8}$ como a restrição de dias e horários de funcionamento. As unidades de saúde, no município de SINOP, funcionam de segunda a sexta-feira, das 7:00 h às 11:00 h, e das 13:00 h às 17:00 h. As estruturas organizacionais são fatores impeditivos para os Enfermeiros realizarem um bom trabalho de promoção da saúde na atenção primária, o que corrobora o estudo realizado na Suécia ${ }^{14}$

Isso representa séria limitação, uma vez que há um intervalo diário descoberto para a prestação de serviço aos idosos, além da possibilidade de sobrecarga de atendimento na única Unidade de Pronto Atendimento (UPA). É necessário fortalecer a "porta de entrada" no nível básico da atenção, demarcando os fluxos dos atendimentos organizados a partir das demandas epidemiológica, sanitária e social $\left.\right|^{5,10}$.

Para implementar essas mudanças, é necessário investimento financeiro para contratação de profissionais e abertura de novas unidades. Com estas modificações, o serviço de saúde, além de ampliar o acesso aos idosos, trará benefícios, principalmente o acolhimento, que possibilita a autonomia, a cidadania e a corresponsabilização na produção do cuidado à saúde 4 .

Em relação ao atributo "longitudinalidade", obteve-se o resultado satisfatório. Resultado satisfatório neste atributo sinaliza que existe continuidade do cuidado em relação ao idoso e o serviço de saúde, com construção de vinculo. Resultados 
positivos foram encontrados em outros estudos, na faixa etária acima de 18 anos $^{11}$ e acima de 60 anos ${ }^{15}$.

O atributo "coordenação" é dividido em coordenação de cuidados e coordenação de informação. Foram avaliados em satisfatórios, apresentando os seguintes resultados $(8,34)$ coordenação de cuidados e $(8,17)$ coordenação de informações. Os itens pior avaliados foram a "contrareferência", "falta à continuidade do cuidado", ou seja, os profissionais encaminham os idosos ao serviço de referência, porém não recebem por escrito qual foi a conduta realizada, o que impossibilita a continuidade do cuidado ao idoso ${ }^{16}$.

Em relação à "disponibilização ao prontuário", os idosos não possuem conhecimento de que podem consultá-lo. Infere-se a necessidade de orientação aos idosos, pois o prontuário é do paciente e o mesmo tem direito de acesso à cópia, pelo código de defesa do Consumidor, e o Código de Ética Médica ${ }^{12,13}$.

O atributo "integralidade" foi elaborado em Serviços Disponíveis e Serviços Prestados. Obtiveram escore superior a 7,0 e foram classificados em satisfatórios. A "integralidade" constitui-se no cotidiano do trabalho por meio das interações que acontecem entre o usuário e serviço ${ }^{17}$. Os idosos atribuíram avaliações insuficientes para as ações de drogas lícitas e ilícitas (serviços disponíveis) e acidentes domésticos, de armas de fogo, de substâncias intoxicantes e de prevenção de queimaduras (serviços prestados).

Os resultados negativos dos itens do atributo integralidade indicam que os profissionais de saúde não realizam as ações a partir da análise da situação de saúde e da realidade local, resultado que foram encontrados em outros estudos ${ }^{10,18}$.

Assim, faz-se aceitável que a gestão da saúde considere a realização de capacitação específica das equipes para esses quesitos, pois a melhoria nesses pontos ineficientes necessita de investimento de baixa complexidade, podendo significar custo/benefício como ação de saúde pública, pois, desta forma, afasta-se a possibilidade de agravos mais dispendiosos ao sistema de saúde ${ }^{19,20}$.

\section{CONCLUSÃO}

Na visão dos idosos, os atributos da APS foram classificados como satisfatórios, sendo o "Acesso de Primeiro Contato" uma barreira a ser transposta para que possam atender às necessidades dos idosos. Nesse sentido, desenvolver ações que beneficiem os idosos, com vistas a favorecer o acesso, através da escuta qualificada, para atendimento das reais necessidades da população idosa, evidencia-se como de grande relevância.

O enfermeiro, como membro da equipe de saúde, pode contribuir de forma expressiva nesse processo. Este estudo aponta para a necessidade de avançar no sentido de oferecer acesso efetivo para o fortalecimento do vínculo entre equipe de saúde e os idosos.

Para isso, o acolhimento, a escuta atenta, o diálogo e o conhecimento da realidade em que o usuário está inserido são primordiais para a o acesso às ações de saúde, o atendimento das necessidades e o envolvimento das famílias na resolução dos problemas e na promoção da saúde ${ }^{21}$.

Como limitação deste estudo, registra-se que a avaliação ocorreu em um único município, o que não permite generalizações para outros contextos.

\section{CONTRIBUIÇÕES DOS AUTORES}

Desenho do estudo. Rosângela Guerino Masochini. Sheila Nascimento Pereira de Farias.

Coleta ou produção dos dados. Rosângela Guerino Masochini. Análise de dados. Rosângela Guerino Masochini. Sheila Nascimento Pereira de Farias. Ana Inês Sousa.

Interpretação dos resultados. Rosângela Guerino Masochini. Sheila Nascimento Pereira de Farias. Ana Inês Sousa.

Redação e revisão crítica do manuscrito. Rosângela Guerino Masochini. Sheila Nascimento Pereira de Farias. Ana Inês Sousa.

Aprovação da versão final do artigo. Rosângela Guerino Masochini. Sheila Nascimento Pereira de Farias. Ana Inês Sousa.

Responsabilidade por todos os aspectos do conteúdo e a integridade do artigo publicado. Rosângela Guerino Masochini. Sheila Nascimento Pereira de Farias. Ana Inês Sousa.

\section{EDITOR ASSOCIADO}

Aline Aparecida Monroe (10

\section{EDITOR CIENTÍFICO}

Ivone Evangelista Cabral (D)

\section{REFERÊNCIAS}

1. Veras R, Parahyba MI. O anacronismo dos modelos assistenciais para os idosos na área da saúde: desafios para o setor privado. Cad Saude Publica.2007;23(10):2479-89. http://dx.doi.org/10.1590/S0102311 X2007001000022.

2. Ministério da Saúde (BR). Secretária de Atenção em Saúde, Departamento de Atenção Básica. Manual do instrumento de avaliação de atenção primária à saúde: Primary Care Assessment Tool PCAtool - Brasil. Brasília: Ministério da Saúde; 2010.

3. Leslie M, Khayatzadeh-Mahani A, Birdsell J, Forest PG, Henderson R, Gray RP et al. Uma história de implementação da transformação da atenção primária à saúde: as redes de atenção primária de Alberta e as pessoas, o tempo e a cultura da mudança. BMC Fam Pract. 2020;21:258. http://dx.doi.org/10.1186/s12875-020-01330-7. PMid:33278880.

4. Starfield B. Atenção primária: equilíbrio entre necessidade de saúde, serviços e tecnologia. Brasília: Unesco; 2002.

5. Assis MM, Jesus WL. Acesso aos serviços de saúde: abordagens, conceitos políticas e modelo de análise. Cien Saude Colet. 2012;17(11):2865-75 http://dx.doi.org/10.1590/S1413-81232012001100002. PMid:23175292.

6. Fracolli LA, Gomes MFP, Nabão FRZ, Santos MS, Cappellini VK, Almeida ACC. Instrumentos de avaliação da Atenção Primária à Saúde: revisão de literatura e metassíntese. Cien Saude Colet. 2014;19(12):4851-60. http:// dx.doi.org/10.1590/1413-812320141912.00572014. PMid:25388193.

7. Instituto Brasileiro de Geografia e Estatística. Censo 2010 [Internet] Rio de Janeiro: IBGE; 2021 [citado 2020 out 27]. Disponível em: http:// censo2010.ibge.gov.br/noticias-censo?busca $=1 \& i d=3 \& i d n o t i c i a=186$ $6 \&$ view=noticia 
8. Gontijo TL, Duarte AGS, Guimarães EAA, Silva J. Avaliação da atenção primária: o ponto de vista de usuários. Saúde Debate. 2017;41(114):74152. http://dx.doi.org/10.1590/0103-1104201711406.

9. Portaria № 2.488, de 21 de outubro de 2011 (BR). Aprova a Política Nacional de Atenção Básica, estabelecendo a revisão de diretrizes e normas para a organização da Atenção Básica, para a Estratégia Saúde da Família (ESF) e o Programa de Agentes Comunitários de Saúde (PACS). Diário Oficial da União, Brasília (DF), 22 out 2011.

10. Araújo LUA, Gama ZAS, Nascimento FLA, Oliveira HFV, Azevedo WM, Almeida Jr HJB. Avaliação da qualidade da atenção primária à saúde sob a perspectiva do idoso. Ciênc saúde coletiva. 2014;19(8):3521-32. http://dx.doi.org/10.1590/1413-81232014198.21862013.

11. Silva SA, Nogueira DA, Paraizo CMS, Fracolli LA. Assessment of primary health care: health professionals' perspective. Rev Esc Enferm USP. 2014;48(Spec No):122-8. http://dx.doi.org/10.1590/S0080623420140000600018. PMid:25517845.

12. Lei $n^{\circ} .8 .078$, de 11 de setembro de 1990 (BR). Código de Defesa do Consumidor. Dispõe sobre a proteção do consumidor e dá outras providências. Diário Oficial da União [Internet], Brasília (DF), 11 set 1990 [citado 2008 jul 4]. Disponível em: http://www.planalto.gov.br/ ccivil_03/Leis/L8078.htm

13. Conselho Federal de Medicina. Código de ética médica: resolução CFM no 1.931, de 17 de setembro de 2009 [Versão de bolso]. Brasília: Conselho Federal de Medicina; 2010.

14. Lundberg $K$, Jong M, Jong MC, Kristiansen LP.Experiências de pacientes no encontro de cuidado na prática de promoção da saúde: um estudo qualitativo na atenção primária à saúde na Suécia. BMC Fam Pract. 2020;21:232. http://dx.doi.org/10.1186/s12875-020-01296-6. PMid:33176696.
15. Costa MA, Alves MTSSB, Branco RMPC, Castro WEC, Ramos CAM Avaliação da qualidade dos serviços de Atenção Primária à Saúde no município de São José de Ribamar, Maranhão, Brasil. Interface Comunicacao Saude Educ. 2020;24(1, supl 1):e190628. http://dx.doi. org/10.1590/interface.190628.

16. Prates ML, Machado JC, Silva LS, Avelar PS, Prates LL, Mendonça EE, et al.. Desempenho da Atenção Primária à Saúde segundo o instrumento PCATool: uma revisão sistemática. Ciênc saúde coletiva. 2017;22(6):1881-93. http://dx.doi.org/10.1590/1413-81232017226.14282016.

17. Fracolli LA, Zoboli ELP, Granja GF, Ermel RC. Conceito e prática da integralidade na Atenção Básica: a percepção das enfermeiras. Rev Esc Enferm USP. 2011;45(5):1135-41. http://dx.doi.org/10.1590/S008062342011000500015. PMid:22031374.

18. Silva AS, Baitelo TC, Fracolli LA. Primary Health Care Evaluation: the view of clients and professionals about the Family Health Strategy. Rev Lat Am Enfermagem. 2015;23(5):979-87. http://dx.doi.org/10.1590/01041169.0489.2639. PMid:26487150.

19. Costa MA et al. Avaliação da qualidade dos serviços de Atenção Primária à Saúde no município de São José de Ribamar, Maranhão, Brasil. Interface Comunicacao Saude Educ. 2020;24(supl 1):e190628.

20. Estrella K, Vianna CM, Sant'Anna AA, Bassan FB. Programa de prevenção de internação para idosos na saúde suplementar: um relato de caso. Rev Bras Geriatr Gerontol. 2009;12(3):497-512. http://dx.doi. org/10.1590/1809-9823.2009.00015.

21. Souza SS. Avaliação dos atributos essenciais da Atenção Básica em Saúde da Criança: Estudo de Caso no Distrito de Saúde Norte da cidade de Manaus-AM [tese]. Manaus: Fundação Oswaldo Cruz; 2016 [citado 2017 set 4]. Disponível em: https://www.arca.fiocruz.br/ handle/icict/31081.

\footnotetext{
${ }^{a}$ Extraído da tese de doutorado intitulada "Qualidade da atenção primária à saúde em um município na região da Amazônia legal na perspectiva de profissionais e idosos", de autoria de Rosângela Guerino Masochini, sob a orientação de Sheila Nascimento Pereira de Farias e co-orientação de Ana Inês Sousa. Programa de Pós-Graduação em Enfermagem, Escola de Enfermagem Anna Nery, Universidade Federal do Rio de Janeiro, em 2017.
} 